\title{
El movimiento estudiantil catalán en el nuevo ciclo de luchas*
}

\author{
The Catalan student movement \\ in the new cycle of struggles \\ O movimento estudantil catalã no novo ciclo de lutas
}

Recibido el 5 de junio de 2014, aceptado el 3 de septiembre de 2014

\author{
Robert González García** \\ México \\ Isabel Benítez Romero*** \\ España
}

, Para citar este artículo:

González García, Robert y

Benítez Romero, Isabel (2014).

El movimiento estudiantil catalán en el nuevo ciclo de luchas. Ánfora, 21(37), 101128. Universidad Autónoma de Manizales. ISSN 0121-6538

\section{Resumen}

Objetivo: analizar la evolución del movimiento estudiantil catalán en los últimos 20 años (1993-2013) para comprender los contextos de implantación de modelos neoliberales de gestión de la universidad y de

\footnotetext{
* Este artículo se enmarca en una investigación sobre el movimiento estudiantil en Europa desde mediados de los noventa a la actualidad. Se aborda, específicamente, el contexto y evolución del mismo en la zona de cultura catalana. Los autores participaron como activistas del movimiento estudiantil catalán (1993-2004 y 2005-2009, respectivamente) y, después, lo estudiaron como investigadores vinculados a la universidad. La mayor parte del trabajo de análisis documental fue realizado entre los años 2008 y 2010, en dos centros de investigación universitarios: el Instituto de Gobierno y Políticas Públicas de la Universidad Autónoma de Barcelona (http://igop.uab.cat/es/) y el Centro de Estudios de los Movimientos Sociales de la Universidad Pompeu Fabra (http://www.upf.edu/moviments/es/).

** Licenciado en Ciencias Políticas y de la Administración y en Sociología, Maestro en iniciación a la investigación en Ciencia Política y Doctor en Ciencias Políticas y de la Administración por la Universidad Autónoma de Barcelona. Profesor-investigador a tiempo completo en la Universidad Autónoma del Estado de Hidalgo. Instituto de Ciencias Sociales y Humanidades, Área académica de Ciencias Políticas y Administración Pública. Correo electrónico: rgonza67@gmail.com

*** Licenciada en Sociología por la Universidad Autónoma de Barcelona. Miembro del Seminario de Economía Crítica Taifa y del Semanario de Comunicación Directa. Colabora en marxismocritico.com. Programadora web en la cooperativa de software libre Projecte Ictineo, creadora de Journal Services sobre Open Journal Systems. Correo electrónico: isabel.benitez.romero@gmail.com
} 
ciclos de movilización a los que responden las diversas etapas del movimiento. Metodología: a partir de la hermenéutica de procesos sociales y a través del análisis de documentación emitida por el movimiento estudiantil catalán y de un ejercicio de reflexión conjunta de los autores con base en datos recogidos de la observación participante y la investigación activista en diversas fases de la movilización estudiantil en Catalunya. Resultados: se presentan cuatro etapas diferenciadas del movimiento estudiantil catalán y cada una de ellas corresponde a cambios en la estructura de oportunidad política del movimiento debidos a respuestas a cambios legislativos de orientación neoliberal o a la emergencia de ciclos de movilización contra la globalización capitalista. Conclusiones: el movimiento estudiantil catalán se ha retroalimentado del ciclo de movilizaciones anticapitalistas, entrando en una dinámica de alianzas crecientes con diversos sectores educativos (laborales y docentes) y de otros movimientos sociales. Sin embargo, el movimiento suele ser repetitivo y reactivo, aunque en algunas fases ha sabido responder a los retos de la internacionalización y la coordinación con otras resistencias al neoliberalismo en el resto de Europa.

Palabras claves: Movimiento estudiantil, Movimiento social, Jóvenes, Globalización, Universidad.

\section{Abstract}

Objectives: to establish the evolution of the Catalan student movement in the last 20 years (1993-2013) in order to understand the implementation contexts of neoliberal models of university management and mobilization cycles that respond to the various stages ofthemovement. Methodology: itis basedonthehermeneutics of social processes, through the analysis of documentation issued by the Catalan student movement and a reflexion exercise of the authors on data collected from participant observation and activist research at various stages of the student movement in Catalonia. Results: four distinct stages of the Catalan student movement are shown. Each one corresponds to changes in the political opportunity structure of the movement due to responses in legislative reforms of neoliberal orientation or to the emergence of mobilization cycles against capitalist globalization. Conclusions: the Catalan student movement has received feedback from the anticapitalist demonstrations cycle, getting involved into a dynamics of growing alliances with various educational sectors (workers and teachers) and other social movements. However, the movement is often repetitive and reactive, although in some phases it has responded to the challenges of internationalization and coordination with other resistance movements to neoliberalism in the rest of Europe.

Keywords: Student Movement, Social Movement, young people, Globalization, University 


\section{Resumo}

Objetivos: estabelecer a evolução do movimento estudantil catalã nos últimos 20 anos (1993-2013) para compreender os contextos de implantação de modelos neoliberais de gestão da universidade e de ciclos de mobilização aos que respondem as diversas etapas do movimento. Metodologia: a partir da hermenêutica de processos sociais e a través da análise de documentação emitida pelo movimento estudantil catalã e de um exercício de reflexão conjunta dos autores com base em dados colheitas da observação participante a pesquisa ativista em diversas fases da mobilização estudantil em Catalunha. Resultados: apresentam se quatro etapas diferenciadas do movimento estudantil catalã e cada uma delas corresponde a câmbios na estrutura de oportunidade política do movimento devidos a respostas em reformas legislativas de orientação neoliberal ou à emergência de ciclos de mobilização contra a globalização capitalista. Conclusões: o movimento estudantil catalã se tem retroalimentado do ciclo de mobilizações anticapitalistas, entrando em uma dinâmica de alianças crescente com diversos setores educativos (laborais e docentes) e de outros movimentos sociais. Porem, movimento algumas vezes pode ser repetitivo e reativo, Contudo em algumas fases tem sabido responder aos retos da internacionalização e a coordenação com outras resistências ao neoliberalismo no resto de Europa.

Palavras chave: Movimento estudantil, Movimento social, Jovens, Globalização, Universidade. 


\section{Introducción}

En 1999, la Huelga de la Universidad Nacional Autónoma de México (UNAM) marcaba un antes y un después en la historia de los movimientos estudiantiles (Rosas, 2001). La Huelga fue para el movimiento estudiantil internacional lo que Seattle para los movimientos contra la globalización capitalista. Seattle y la Huelga, además, no se pueden entender sin el levantamiento zapatista de 1994, en los albores de la crítica al neoliberalismo y a sus instituciones (Banco Mundial, FMI, TLC, ALCA, UE, etc.).

Por su parte, el movimiento estudiantil catalán ${ }^{1}$ se configura como respuesta a los cambios en la estructura de oportunidades políticas: cambios legislativos, cambios de gobierno, ciclos de movilización o emergencia de otros potentes movimientos, situación internacional y división entre las élites dirigentes (Mc Adam, 1998; Brocket, 1991; Kitchlet, 1986; Tarrow, 1997 y Tilly, 1991). Destaca el peso de factores de oportunidad como el ciclo de luchas del movimiento contra la globalización capitalista, los cambios legislativos $\left(\mathrm{LOU}^{2}, \mathrm{LEC}^{3}, \mathrm{LOMCE}^{4}\right.$ y construcción del EEES $^{5}$ ), la situación internacional (Guerra de Iraq en 2003 o la primavera árabe en 2011) y la rearticulación de la izquierda alternativa, de la izquierda independentista y de los movimientos sociales (como el de los okupas y el $15 \mathrm{M})$.

Respecto a los ciclos de movilización, se coincide con los autores que destacan tres grandes ciclos de protesta en la historia contemporánea de las sociedades occidentales: un primero desde 1848 hasta la segunda guerra mundial, con el protagonismo del movimiento obrero; un segundo, protagonizado por los llamados nuevos movimientos sociales (ecologismo, pacifismo, segunda ola del feminismo) situado entre 1968 y 1977, y un tercero -desde mediados de los 90que se ha popularizado como "movimiento antiglobalización", pero que otros

\footnotetext{
1. Entendemos catalán en un sentido amplio, integrando a los territorios conocidos como Països Catalans (Catalunya, País Valencià, Illes Balears, Catalunya nord -bajo jurisdicción francesa- y Franja de Ponent). La propia vertebración nacional del movimiento estudiantil catalán hace imprescindible la apertura del enfoque territorial.
}

2. Ley Orgánica de Universidades de 2001, a través de la cual se legaliza el modelo neoliberal de gestión de la universidad española (Camargo, 2001).

3. Llei Catalana d'Educació de 2009 qué desarrolla al máximo las competencias educativas del gobierno catalán y profundiza en el modelo neoliberal de gestión educativa (Bonal y Verger, 2013)

4. Ley Orgánica para la Mejora de la Calidad Educativa de 2013, también conocida como Ley Wert, por el apellido del ministro derechista de educación. Se trata de un giro neo-conservador y neo-centralista del sistema educativo (Salazar-Alonso, 2012 y González, 2013).

5. Espacio Europeo de Educación Superior, ámbito de organización educativo iniciado en 1999 con el proceso de Bolonia qué homogeneíza desde una orientación neoliberal las universidades europeas (Carreras, Sevilla y Urbán, 2006). 
prefieren denominar nuevos movimientos globales o altermundistas (Tarrow, 1997; Calle, 2005; Herreros, 2004). A éste ciclo es al que se hace referencia con el título del artículo.

También es conveniente aclarar qué se entiende por ciclo de movilización: siguiendo a Mc Adam (1998) y Tarrow (1997) se puede definir ciclo de protesta como

Una fase creciente de conflicto y de enfrentamiento a todo el sistema social, que conlleva los siguientes rasgos: (i) rápida difusión de la acción colectiva, desde los sectores tradicionalmente con mayor capacidad de movilización hacia los sectores con menos capacidad; (ii) aceleración de las pautas de innovación en el repertorio de acción colectiva; (iii) combinación de participación contenida y participación transgresora, con la creación de nuevos movimientos sociales; (iv) y finalmente, secuencias de interacción intensificada entre los grupos desafiantes y las autoridades, que finalmente pueden culminar en revueltas, en reforma, en represión y, a veces, en revolución (Herreros, 2004, p. 4).

Por otro lado, la literatura académica coincide en señalar que la crisis del petróleo de 1973-74 supuso el inicio de una larga ola de recesión de la acumulación capitalista y la excusa perfecta para el surgimiento de las teorías neoliberales ${ }^{6}$. Las privatizaciones, la desregulación del mercado laboral y la reducción del gasto público, serán las medidas que empiezan a aplicar los diversos gobiernos con diferentes ritmos y formas en función de las circunstancias geopolíticas de cada región (Etxezarreta, 2001). Por otra parte, y como consecuencia no querida del ciclo de luchas 68-77, en los países centrales del capitalismo, la fábrica empieza a difuminarse como lugar de producción por excelencia, para dar paso a un modelo pos-fordista de producción. En tal modelo, la flexibilidad, versatilidad y movilidad de la mano de obra sirven al capital para disminuir la conflictividad obrera y continuar con la apropiación de la plusvalía (Rodríguez, 2003). Estos profundos cambios estructurales, se traducen en la universidad con el tránsito del modelo de universidad de masas al de universidad empresa. Las características específicas de este nuevo modelo de universidad (Sevilla, 2006) no son el objeto de este artículo, pero fundamentan las movilizaciones estudiantiles ya desde los años ochenta, y cada vez de forma más clara en los noventa y primera década del dos-mil contra la mercantilización de la educación.

En cuanto a la relevancia del presente artículo, hay que señalar que el estudio de los movimientos estudiantiles en el Estado español es un fenómeno reciente en la literatura académica. Existen algunos estudios sobre el movimiento estudiantil de la segunda etapa del franquismo y la transición (Navarrete,

6. Si la tasa de beneficio del ciclo expansivo de posguerra se estaba reduciendo, la receta sería expandir la mercancía a aquellos ámbitos que habían sido ganados por el movimiento obrero en el pacto keynesiano. 
1995), de posteriores ciclos de movilización estudiantil como el del 1986-1987 (Vaquero, 2004; Rodríguez, 2003) y libros más relacionados con el activismo (Colectivo Maldeojo, 2001). Pero no es hasta bien entrados en el siglo XXI, cuando algunos investigadores empiezan a explicar las respuestas estudiantiles al denominado proceso de Bolonia (Carreras, Sevilla y Urbán, 2006, Fernández, 2014), poniendo el acento en las resistencias al proceso de mercantilización de la enseñanza universitaria.

Otro de los objetivos de este trabajo es que el mismo sea útil para el movimiento. De esta manera, esta investigación no se diseñó para saturar el tema de estudio, sino que quiere ser una prospección y un análisis a discutir con los protagonistas -la mayoría de ellos militantes estudiantiles- pero también con las autoridades universitarias, a menudo atrapadas en la agenda gubernamental de las grandes instituciones transnacionales. Por esta razón, se han realizado retornos a los sectores involucrados en forma de charlas, ponencias o este mismo artículo, más dirigido a la comunidad académica.

Finalmente, el objetivo principal de este artículo es analizar, interpretar y explicar las diversas etapas del movimiento estudiantil catalán de los últimos 20 años (1993-2013), entendidas como respuestas contra la progresiva implantación de este modelo de universidad-empresa. También se busca estudiar las alianzas con otros movimientos y su inmersión en ciclos de movilización más amplios contra la globalización capitalista - primero- y contra las políticas de austeridad presupuestaria emprendidas por los gobiernos europeos como respuesta a la crisis de 2008 -después-. Esta investigación colabora con el esfuerzo de otros autores para explicar este fenómeno, al tiempo que pretende aportar algo de memoria histórica al movimiento estudiantil catalán.

\section{Metodología}

Este artículo, como producto de un trabajo de campo dilatado, adopta a menudo la forma de crónica e interpretación de los acontecimientos más relevantes en la historia reciente del movimiento estudiantil catalán y sus resistencias al proceso de mercantilización de la universidad pública. La metodología utilizada es cualitativa y entronca con las tradiciones de la observación participante e investigación activista (Verger, 2005). Los autores del texto han formado parte del movimiento estudiantil en diversas etapas, lo que les ha permitido tener acceso directo a las fuentes y participar en todos los eventos analizados con el tamiz de la teoría social y la reflexión académica. Posteriormente, desde su posición de profesores-investigadores, han participado en las convocatorias públicas del movimiento o en los espacios de confluencia entre estudiantes y magisterio 
movilizado. Las notas de campo y las propias agendas de los investigadores han permitido recoger multitud de datos etnográficos que permiten (re)escribir esta historia del movimiento estudiantil catalán.

En segundo lugar, el enfoque metodológico para el estudio de un movimiento social como el estudiantil no puede ser distante y frío, ya que este tipo de actor colectivo no se puede conocer de esta manera. Así que queremos explicitar. Por eso, aquí se explica el doble propósito de esta investigación, por un lado el conocimiento del movimiento estudiantil universitario catalán de los últimos 20 años y, por el otro, la participación en sus actividades y la observación a fondo de su funcionamiento. Al mismo tiempo, se ha tratado de mantener una observación participante de ángulo abierto, con el propósito añadido de estudiar los aspectos políticos tácitos en el funcionamiento del movimiento (formas organizativas, liderazgos, discursos, contexto cultural). El hecho de los autores haber sido miembros del movimiento, en un periodo, e investigadores, después, ha ayudado a asegurar una experiencia desde dentro y fuera de la escena, en la doble condición de miembros y espectadores.

Finalmente, el análisis documental de prensa de la época y de materiales del propio movimiento estudiantil ha sido complementario. En concreto, se ha utilizado la técnica del análisis del contenido de los discursos del movimiento estudiantil, especialmente a través de sus manifiestos, documentos, pasquines y consignas, comparándolo con la "opinión publicada" (mediante el análisis de las noticias sobre el movimiento en los medios masivos) y con los textos producidos por las autoridades universitarias (Juntas de gobierno, equipos rectorales, etc.). De esta forma, se buscó comprender las diferentes estrategias discursivas en cada periodo histórico, que develan la pugna simbólica entre dos relatos: el de las autoridades universitarias, el cual argumenta que los procesos vividos forman parte de la modernización de la universidad catalana y el del movimiento estudiantil, que denuncia los procesos de privatización y mercantilización de la enseñanza pública universitaria. Ahora bien, estos discursos han variado con cada etapa, respondiendo a las coyunturas y estructuras de oportunidad política de cada momento -como se aprecia en los resultados-. Por ejemplo, los discursos del movimiento estudiantil se han correspondido en cada momento con los ciclos de movilización a nivel global. En las conclusiones se comprueba que cada etapa o ciclo del movimiento estudiantil se enmarca en una etapa o ciclo de los movimientos contra la globalización neoliberal. Y un indicador de esto ha sido observar el uso de categorías como "mercantilización", "democracia participativa”, "dignidad", "justicia" en los discursos de ambos movimientos. Asimismo, la revisión de la bibliografía sobre procesos de mercantilización de la educación en España y Europa, así como la referente a los movimientos estudiantiles que se han opuesto a los mismos, ha sido la fuente indispensable para orientar el análisis. 


\section{Resultados}

El movimiento estudiantil universitario catalán de las dos últimas décadas ilustra un giro progresivo desde movilizaciones corporativo-estudiantiles en contra del alza de las tasas de matrícula, hacia campañas de identificación y denuncia del proyecto mercantil contra la enseñanza pública superior. En ese trayecto el movimiento se ha aproximado y ha establecido vínculos con otros sectores intrauniversitarios (personal de administración y docentes) y extrauniversitarios (movimiento obrero, movimiento okupa y antiglobalización).

Precisamente, la riqueza y continuidad del tejido organizativo, social y político de Catalunya -sostenida desde la Transición a la Democracia- encuentran su reflejo en el movimiento estudiantil catalán, donde se registra un conjunto de anomalías harto peculiares: la hegemonía en la representación institucional de las asambleas de facultad en la segunda universidad en número de estudiantes de Catalunya (la Universitat Autònoma de Barcelona); la perduración en el tiempo de estructuras asamblearias interuniversitarias (Plataforma Mobilitzadora en Defensa de la Universitat Pública, Coordinadora d'Assemblees d'Estudiants) y la existencia, desde 2006, de un sindicato de estudiantes con una implantación territorial y cuantitativa sin equivalente en el resto del Estado, el Sindicat d'Estudiants dels Països Catalans (SEPC).

La suma de estos factores internos y los factores de oportunidad de las coyunturas políticas, española y catalana, están en la base de un ciclo de movilizaciones con un impacto social de agitación y movilización superior al del resto del Estado español. De esta forma, en los resultados se realizará una descripción y un análisis de los últimos 20 años de movimiento estudiantil donde se identifican cuatro periodos o sub-ciclos. En cada uno de ellos se desglosa la vertebración de las resistencias estudiantiles y sus sinergias con el ciclo internacional de luchas.

Así, la primera etapa (1993-1999) comprenderá las movilizaciones contra las tasas de matrícula y la aplicación real de la reforma universitaria post-franquista (Rodríguez, 2003). Coincidirá, a nivel del Estado español, con gobiernos en minoría de PSOE (1993-1996) y PP (1996-2000) con pactos con los partidos nacionalistas de centro-derecha (CIU, PNV y CC) ${ }^{7}$. Esta también es la etapa de gestación del movimiento antiglobalización con las campañas de 50 años bastan contra el FMI y el BM, las Euromarchas contra el Paro y la Precariedad o los Encuentros Intergalácticos por la Humanidad y contra el Neoliberalismo, como hitos fundamentales. El segundo periodo (2000-2004) comprende un ciclo completo que va de los precedentes y movimiento estudiantil contra la LOU,

7. Convergència i Unió (catalán), Partido Nacionalista Vasco y Coalición Canaria. 
hasta el protagonismo estudiantil en las movilizaciones contra la guerra de Irak en 2003. Coincide este periodo con la eclosión definitiva del movimiento antiglobalización en el Estado español, así como con un ciclo creciente de movilizaciones contra las políticas derechistas y autoritarias del gobierno con mayoría absoluta del Partido Popular (Calle, 2005; González, 2011), que culminan con la caída sorprendente del gobierno de Aznar en las elecciones del 14 de marzo de 2004 después de una insólita movilización contra las mentiras del gobierno sobre los atentados del 11M en Atocha (Sampedro, 2005).

La tercera etapa (2005-2010) aborda los precedentes y eventos centrales del ciclo de movilización anti-Bolonia, contra la implantación del Espacio Europeo de Educación Superior por su orientación neoliberal (Fernández, 2014). Este ciclo coincide con cierto reflujo de los movimientos sociales ante la victoria del centro-izquierda y, en especial, por las políticas progresistas del primer gobierno Zapatero (2004-2008). Aun así, la crisis económica de 2008 y el abordaje en términos de recortes sociales del segundo gobierno socialista (2008-2011) provocarán una lenta revitalización de las movilizaciones populares, que tendrá su máxima expresión en el periodo subsiguiente. Finalmente, la última etapa -ya en plena crisis económica y todavía en curso- es analizada por algunos autores como una verdadera revolución democrática que tiene su inicio en el movimiento de los indignados del 15 de mayo de 2011 y su referente internacional en la Primavera Árabe (Calle, 2012; Ibarra, 2012; Rodríguez, 2013). En esta etapa la protesta social se focaliza contra las políticas de austeridad y de recortes impulsadas tanto por el segundo gobierno Zapatero, como por el del PP de Rajoy (2011-2015) en el conjunto del Estado y por los gobiernos de CIU en Catalunya. El movimiento estudiantil catalán se moviliza contra los recortes en educación, la Estrategia Universidad 2015 y la implementación de nuevas leyes educativas de corte neoliberal, como la LEC o la LOMCE (Fernández, 2014).

\section{3-1999, movilizaciones anti-tasas: inicios del nuevo ciclo}

Desde las grandes manifestaciones contra la Ley para la Reforma Universitaria y contra la selectividad en los años ochenta, el movimiento estudiantil no volvió a revitalizarse como movimiento de masas hasta la "resaca del 92" . Éste, es también el momento de la etapa de gestación del ciclo contra la globalización capitalista, que en Catalunya estará protagonizado por el movimiento okupa, el movimiento antimilitarista y el movimiento de solidaridad internacional. Así, desde el otoño del 93 hasta primavera del 94 se generalizaban las protestas

8. Expo Sevilla '92 y las manifestaciones de repulsa a la operación urbanística y a la celebración del $\vee$ Centenario. Las Olimpiadas Barcelona '92 y la Operación Garzón, en la que se aplica la Ley Antiterrorista a sesenta militantes independentistas que son encarcelados y torturados. 
contra una subida de tasas de matriculas cinco puntos por encima de la media estatal. Entre los estudiantes de la época que reactivaban las asambleas era fácil encontrar activistas de los principales centros sociales okupados de Barcelona, de los colectivos por la insumisión al servicio militar y a la Prestación Social Substitutoria o brigadistas internacionales que veían en la Selva Lacandona mexicana la nueva esperanza de lucha. En la UAB la movilización condujo a la okupación del rectorado, que sería desalojado por un impresionante despliegue de los recién estrenados cuerpos represivos autóctonos (los Mossos d'Esquadra).

En estos momentos, las asociaciones estudiantiles en Catalunya se caracterizaban por sus fuertes vínculos con los partidos políticos institucionales. Así, la más histórica de ellas, la FNEC (Federació Nacional d'Estudiants de Catalunya), actuaba como escuela de cuadros de Convergència $i$ Unió, partido hegemónico en esos momentos en Catalunya. Por su parte, la AJEC (Associació de Joves Estudiants de Catalunya), hacía lo propio con el PSC ${ }^{9}$. Ambas conservaban una representación desproporcionada en los órganos -con respecto a su peso real en el movimiento- y ya desde finales de los 80 se estaban viendo superadas por otras dos más a la izquierda: la AEP (Associació d'Estudiants Progressistes) ${ }^{10}$ y el BEI (Bloc d'Estudiants Independentistes $)^{11}$. Por último, en un plano más marginal, aunque con cierta implantación en facultades de Derecho, las asociaciones próximas al Partido Popular y a la extrema derecha, OCEU (Organización Catalana de Estudiantes Universitarios) y AU (Alternativa Universitaria), completaban una mapa político frente al que emergió con fuerza un nuevo movimiento asambleario.

En la revitalización del asamblearismo jugaban un papel central dos procesos de la intrahistoria del movimiento estudiantil de la UAB. En el curso 93/94, un grupo de estudiantes de diversa procedencia ideológica (independentistas revolucionarios, comunistas, autónomos y ácratas) retomaron la asociación Alternativa Estel, con el objetivo de actuar de nexo entre los movimientos sociales de la época y la universidad, así como la potenciación de las asambleas de facultad, que si bien existían desde los años 70, habían perdido fuerza.

El segundo proceso fue el nacimiento de la Coordinadora de Asambleas de Facultad (CAF) durante ese mismo curso académico, tras la okupación del rectorado en protesta por la subida de tasas y ante la necesidad de superar el marco de unos sindicatos estudiantiles pactistas y que no representaban el sentir

9. Partit dels Socialistes de Catalunya, referente catalán del PSOE (Partido Socialista Obrero Español)

10. Asociación próxima al partido eco-socialista ICV (Inciativa per Catalunya-Verds), pero sobre todo a las juventudes de los partidos comunistas tradicionales (PSUC y PCC).

11. Con mayoría de militantes de las juventudes de ERC (Esquerra Republicana de Catalunya). 
de la mayoría del estudiantado movilizado ${ }^{12}$. El funcionamiento de la CAF serviría de modelo para otras universidades (también fuera del territorio catalán). La horizontalidad en la toma de decisiones y la democracia directa sería su característica fundamental. Las propuestas emanaban de las asambleas semanales de facultad y los jueves se reunían los portavoces de cada una de ellas para coordinarlas y repartir tareas. Otra característica importante de la CAF es que serviría de marca electoral del movimiento estudiantil (real). A diferencia de otros movimientos asambleístas, el de la UAB no cedería la representación estudiantil en los órganos de gobierno de la universidad a las asociaciones estudiantiles tradicionales. En pocos años, las asambleas se convertirían en las listas más votadas en todo tipo de elecciones (de facultad, de claustro general, etc. ${ }^{13}$.

También en este primer periodo, se dio una dinámica unitaria del movimiento estudiantil. De esta forma, en 1995 se fundó la PMDUP (Plataforma Movilitzadora en Defensa de la Universitat Pública $)^{14}$. La PMDUP hacía un análisis de la situación de la universidad en Catalunya y daba a conocer sus cuatro objetivos principales: a) la reivindicación de la enseñanza pública; b) la defensa de la igualdad de oportunidades a la hora de acceder a la educación superior; c) defender un concepto de calidad universitaria que considerase la dimensión humana y formativa del sistema universitario y d) la necesidad de que toda la sociedad participase en la definición del papel de la universidad pública.

La estructura de la plataforma pretendía ser asamblearia de base y funcionar a partir de delegados rotativos. Las decisiones se tomarían por consenso o por amplia mayoría, lo cual reforzaba la unidad del movimiento y multiplicaba su capacidad de incidencia. Los sindicatos estudiantiles no debían -en principiodefender posicionamientos como organización, sino que sus miembros debían participar en las asambleas al mismo nivel que el resto de estudiantes no asociados.

En definitiva, en el curso 93/94 confluyeron diversos factores de oportunidad permitiendo la aparición de un nuevo ciclo y de una nueva generación militante. Las movilizaciones de los cursos subsiguientes, tendrían en las progresivas alzas

12. La ocupación del rectorado se realizó con la oposición -incluso física- de los líderes sindicales estudiantiles, y durante la misma, se debatió la necesidad de autorganizarse al margen de esas estructuras tan próximas a los partidos. Aun así, los sindicatos de izquierdas, AEP y BEI rectificarían sobre la marcha y se unirían a la dinámica asambleísta.

13. Todavía en 2009, los portavoces de la CAF eran el $80 \%$ de la representación estudiantil en el claustro general de la UAB, aunque en 2012 esta representación bajó al 58\% (http://www.uab.es/ Document/302/676/Resultats_definitius_Sector_C_ELECLAUSTRE2012,0.pdf.) Se incluyen los 12 representantes de la Asamblea de Jóvenes Investigadores, que a pesar de no formar parte de la dinámica organizativa de la CAF (pues son estudiantes de Máster y Doctorado) han mostrado históricamente su afinidad con la misma (González, 2011).

14. El Manifest de la Campanya en Defensa de la Universitat Pública contó con el apoyo de todos los estamentos universitarios y fue aprobado por los claustros de la UAB y de la UPC. 
de tasas el leitmotiv para la movilización de masas a favor de una universidad pública; pero también destacarían las luchas contra el inicio de la privatización -mediante "externalización"- de los servicios de transporte, fotocopias o restauración. Asimismo, se establecerían fuertes vínculos con los primeros eventos de la "antiglobalización", como la campaña 50 años Basta contra el Banco Mundial y el FMI, las euromarchas contra el paro y la precariedad o las primeras expediciones a las contracumbres de la UE (Antentas, 2001). Estos mimbres organizativos constituyeron el espacio y prácticas que serían puestas a prueba en el momento de agudización de las presiones sobre la universidad pública.

En marzo de 2000, la victoria con mayoría absoluta del PP, permitió a Aznar desplegar el programa neoliberal, neoconservador y autoritario de la derecha española, pero desató una oleada de protestas por temas diversos (contra el trasvase del río Ebro en Catalunya, contra la gestión de la catástrofe ecológica del Prestige en Galicia, contra las medidas lesivas de los derechos de los migrantes, etc.) al tiempo que el movimiento contra la globalización capitalista vivía su etapa de eclosión con multitudinarias movilizaciones como la Campaña contra el BM de 2001, la Campaña contra la Europa del Capital en 2002 y las posteriores y enormes manifestaciones contra la Guerra de Irak en 2003. Pero esto ya forma parte de la siguiente etapa.

\section{0-2004, del "No a la LOU al "No a la guerra": vínculos con las resistencias anticapitalistas}

Como consecuencia de lo expuesto en el apartado anterior, este es el periodo que muestra una movilización más masiva y generalizada al coincidir con el momento más álgido del movimiento altermundista en Catalunya, así como con el inicio de un ciclo de movilizaciones sociales de todo tipo contra las políticas del gobierno del Partido Popular. También fueron los años de puesta en escena de una nueva generación de activistas y militantes universitarios y de un nuevo discurso anti-mercantil en torno a las protestas contra el Informe Universidad 2000 (o Informe Bricall), la Ley Orgánica de Universidades (LOU) y, posteriormente, la visualización de la construcción del Espacio Europeo de Educación Superior (EEES) (Carreras, Sevilla y Urbán, 2006).

La filtración del Informe Bricall -que no era sino una propuesta de "modernización" del sistema universitario que situaba a la universidad española en el tránsito de la universidad de masas a la universidad-empresa (Sevilla 2006 y 2010) - catalizaría protestas y paros estudiantiles en todo el Estado en el segundo semestre del curso 1999/2000. En Catalunya, el 23 de marzo de 2000, la manifestación reunió alrededor de 11.000 estudiantes (El Mundo, 24/03/2000). En general, la asistencia a las manifestaciones no dejó de crecer desde entonces, y el movimiento se adaptó tácticamente a los cambios legislativos que se 
avecinaban. Así, de la oposición al Informe Bricall se pasó a la lucha contra la LOU, ya que ésta recogía casi todas las propuestas mercantilizadoras del mismo.

La recta final de la lucha contra la LOU no puede entenderse sin el Foro Universitario Alternativo (FUA), el cual representaba la sintonía del movimiento con el nuevo ciclo de luchas contra la globalización y la primera experiencia de coordinación estatal del movimiento estudiantil real. Se prescindía, así, de las burocracias estudiantiles (León y Sanllorente, 2008). Reunido en Valladolid en julio de 2001, además de suponer un encuentro de crítica a la universidad existente y debate de los proyectos de universidad crítica y popular, el FUA estableció un ambicioso calendario de movilizaciones contra la LOU en el ámbito estatal, partiendo de las bases del movimiento real: coordinación desde abajo (aunque sea más lenta) y diversidad de tácticas en lo territorial. Es significativo que por la parte catalana acudieran al encuentro los tres sectores más importantes del movimiento: la CAF, como representante del sector asambleísta, la AEP y la CEPC ${ }^{15}$.

La filtración del proyecto de ley daba el pistoletazo de salida para las luchas estudiantiles en otoño de 2001, las cuales darían un salto cualitativo en su reivindicación -ya no contra medidas parciales, sino contra todo el proyecto: (la Ley Orgánica de Universidades) - y cuantitativo en su capacidad de convocatoria. Este hecho fue utilizado como ariete por el oportunismo opositor del PSOE contra el PP. Así, el PSOE y su lobby mediático (grupo Prisa) agitaron las iras durante un trimestre, mientras los estudiantes tomaron facultades, calles y campus sin apenas oposición de los rectorados y con las simpatías abiertas de las burocracias sindicales de CCOO y UGT ${ }^{16}$.

Las movilizaciones contra la LOU que se desarrollaron durante el primer semestre del curso 2001-2002 fueron masivas. Tal y como se había acordado en el FUA de Valladolid, los días 13 de noviembre -en Catalunya- y 14 -en el resto del Estado-, todas las universidades públicas españolas fueron a la huelga y más de 200.000 estudiantes se movilizaron en las mayores manifestaciones estudiantiles desde mediados de los ochenta (La Vanguardia, 2001). En Santiago de Compostela, la huelga duró casi seis meses. A ella se unió el profesorado precario por lo que se convirtió en la punta de lanza de un movimiento estudiantil que también tuvo especial fuerza en Madrid y Sevilla. Por su parte, en Catalunya, hubo huelgas desiguales en función de la universidad e incluso de las facultades. La UAB - que llegó a paralizarse durante dos semanas- y la Universitat de Girona se declararon insumisas a la LOU en sendos claustros en noviembre de $2001^{17}$.

15. Coordinadora d'Estudiants dels Països Catalans, un nuevo sindicato estudiantil fundado en 2000 de la fusión de sindicatos independentistas de Catalunya, País Valencià y les Illes Balears (CEPC, 2006).

16. Comisiones Obreras y Unión General de Trabajadores son los sindicatos mayoritarios en España.

17. Posteriormente, las maniobras de los respectivos equipos de gobierno de ambas universidades dejaron sin efecto los acuerdos de claustro. 
El movimiento huelguista, que alcanzó altos grados de auto-organización, desarrollo de acciones originales y contundentes (performances, okupaciones, huelgas de consumo, jornadas de lucha, docencia alternativa, etc.), así como decenas de medios de contra-información (pasquines, boletines, carteles, incipientes páginas web y listas de correo electrónico), empezó su declive en la Marcha sobre Madrid del 1 de diciembre. La convocatoria de una manifestación oficialista que reuniría a 200.000 personas ${ }^{18}$, encabezada por el entonces líder del PSOE, José Luís Rodríguez Zapatero, generó gran malestar en el movimiento. Una parte de los activistas convocaron una manifestación alternativa y ocuparon durante un fin de semana la Universidad Complutense de Madrid. Más de mil activistas estudiantiles catalanes acudieron a la manifestación alternativa, que fue invisibilizada y reprimida; mientras que la oficialista ponía punto final al movimiento con la promesa de derogación de la LOU por parte de Zapatero ${ }^{19}$.

Los vínculos del movimiento estudiantil con los nuevos movimientos globales serían más fuertes en la confluencia en las luchas que en el ámbito organizativo. Aun así, algunos nuevos colectivos, como el MRG-UAB ${ }^{20}$ o la XUSI ${ }^{21}$, sí vinculaban de forma orgánica ambos movimientos, al tiempo que servían de elementos de coordinación de los mismos y de transmisión de memoria histórica entre dos generaciones militantes. En estos colectivos estudiantiles, así como en algunas asambleas de Facultad, se producía el encuentro entre los militantes de los 90 y la nueva generación surgida en 2000. Por otro lado, la presencia de estudiantes universitarios en las principales citas altermundistas fue fundamental (González y Barranco, 2007). El discurso contra la LOU, que emanaba de los manifiestos y resoluciones de los encuentros estudiantiles de la época, se vinculaba a la lucha contra los organismos internacionales que suelen servir de ariete a este tipo de reformas regresivas, como la OMC o la propia UE (Camargo, 2001).

En 2002, una vez aprobadas la LOU y su referente catalán, la Llei d'Universitats de Catalunya (LUC), la ventana de oportunidad del cambio legislativo parecía cerrarse momentáneamente, sobre todo por la sensación de derrota y de engaño. En las facultades comenzaban a introducirse los primeros másteres adaptados al EEES, las tasas subían invariablemente por encima de la inflación y se articulaban los primeros "planes piloto" de grado en algunas universidades. Paradójicamente, durante estos años, el movimiento estudiantil catalán parecía más interesado en lo que pasaba "fuera" de la universidad que en su interior. A pesar de ello, unos cuantos activistas seguían denunciando, en charlas y talleres

18. Datos de los organizadores. La Delegación del Gobierno cifró en 55.000 (El Mundo, 2/12/2001).

19. Promesa, que -como recordarán los anti-Bolonia en 2009-incumplió.

20. Movimiento de Resistencia Global de la UAB

21. Xarxa Univeristària de Suport als Immigrants (Red universitaria de apoyo a los inmigrantes) 
de formación, el proceso de mercantilización de la universidad, ahora asociado al nombre de una ciudad italiana: Bolonia.

Las movilizaciones estudiantiles más importantes de este periodo se produjeron en el contexto del gran movimiento social contra la intervención española en la Guerra de Irak ${ }^{22}$. El 20 de marzo de 2003, día de inicio de los ataques aéreos de la coalición aliada sobre la población iraquí, todas las universidades catalanas fueron a la huelga, protagonizando manifestaciones espontáneas por todo el territorio. La más espectacular fue la de miles de estudiantes de la UAB, que caminaron 25 kilómetros por la autopista hasta la ciudad de Barcelona. Esta misma universidad se proclamó en huelga indefinida, a través de un comunicado de la asamblea de Campus, en el que era visible un claro relevo generacional con respecto al movimiento contra la LOU. Los estudiantes también fueron los protagonistas de los espacios de desobediencia civil en el movimiento antiguerra en Barcelona ${ }^{23}$.

Durante estos dos años, se celebraron dos Foros Sociales Europeos en París y en Londres, a los que acudieron miles de universitarios organizados por el nodo universitario de la Xarxa de Mobilització Global (Red de Movilización Global). Centenares de activistas estudiantiles pudieron compartir experiencias con activistas de los distintos países del viejo continente, tomando conciencia del carácter internacional de los procesos de mercantilización de la enseñanza y señalando al proceso de Bolonia como blanco de las movilizaciones estudiantiles del próximo periodo.

Por lo que se refiere al cierre de esta etapa, el año 2004 marcó el inicio de cierto declinar en el movimiento contra la globalización y la guerra, que de alguna manera murió de éxito. Efectivamente, los atentados del 11 de marzo de 2004 y la gestión de los mismos por parte del gobierno del PP desataron una audaz movilización popular que ha sido producto de diversos estudios y análisis (Sampedro, 2005; Iglesias, 2005). El 13 de marzo, miles de personas desafiaron la prohibición de manifestarse en la jornada previa a unas elecciones generales y se concentraron en las sedes del partido del gobierno de todas las capitales de provincia y ciudades importantes. Al día siguiente -contradiciendo todas las encuestas pre-electorales-, el PSOE de Zapatero ganaba las elecciones por mayoría absoluta y su primera medida al tomar el poder - la retirada de las tropas españolas de Irak- daba una victoria sin precedentes a los movimientos sociales al tiempo que impactaba en cierto reflujo de los mismos. Sin embargo, el nuevo gobierno no derogó la LOU sino que le dio un suave maquillaje que no cambió su orientación neoliberal. Pero esa realidad no se hubiera desvelado sin

22. Para un buen análisis de las movilizaciones análogas en Vizcaya, ver García y Fernández (2008).

23. En las tres acampadas de protesta que durante dos meses se establecieron de forma permanente ante las sedes del PP, el Gobierno Civil y los gobiernos municipal y autonómico. 
el movimiento estudiantil que protagonizó la siguiente etapa y que se analiza en el apartado siguiente (Fernández, 2014).

\section{5-2010, el ciclo anti-Bolonia}

Este tercer periodo está marcado por el incremento en la intensidad del enfrentamiento entre estudiantes críticos con la deriva neoliberal de la universidad y autoridades universitarias y gubernamentales, partidarias o sumisas a la misma. Es por esto que esta etapa se puede considerar como un ciclo en sí misma. También se caracteriza por la internacionalización del movimiento que de su anterior movilización contra una ley estatal (la LOU), transitará, paulatinamente, hacia la crítica al modelo europeo de universidad (el Proceso de Bolonia o el Espacio Europeo de Educación Superior).

Así, mientras se percibía cierta pérdida de protagonismo de los movimientos antiglobalización en Catalunya -con una disminución de la participación en contracumbres y Foros Sociales internacionales- el ciclo de luchas estudiantiles se extendía por el viejo continente: otoño de 2005 en Italia (reformas Moratti y Zecchino- Berlinguer, aplicación de Bolonia); noviembre de 2005 y mayo de 2006 en el Estado español (sendas manifestaciones contra el Proceso de Bolonia); diciembre de 2005 en Dinamarca (contra la prohibición de exámenes universitarios colectivos); mayo de 2006 en Francia (okupaciones, huelgas y manifestaciones contra el Contrato Primer Empleo, CPE) y en Grecia (contra la ley de educación universitaria) (Sevilla y Urbán, 2008).

A partir del curso 2005/2006, se inició una dinámica de convergencia de las movilizaciones estudiantiles a nivel europeo. El día 17 de noviembre de 2005 miles de estudiantes salieron a la calle en Barcelona, Madrid, Roma y Hamburgo en contra el proceso de Bolonia ${ }^{24}$. En años sucesivos, el 17 de noviembre -día internacional del estudiante- se repetirían las manifestaciones. La confluencia del movimiento europeo se fraguaría en las reuniones internacionales de estudiantes en Bakaiku (marzo de 2006), París (octubre de 2006) y Atenas (octubre de 2007)25.

En este periodo, la vanguardia estudiantil orientaba el esfuerzo analítico hacia el marco general europeo, el acuerdo de la OMC de liberalización de mercados de servicios y la Estrategia de Lisboa. En los campus, la actividad estudiantil se concentraba en campañas sectoriales que referían a este marco como consigna no interiorizada ("No a Bolonia”): defensa de la lengua catalana, contra el catálogo de titulaciones, etc. Se trataba de una denuncia genérica del EEES desvinculada de las prácticas cotidianas.

También en este periodo, la PMDUP entró en crisis. El reflujo de las

24. La de Barcelona se saldó con decenas de heridos y 46 detenidos.

25. Las dos últimas bajo el nombre de Foro Europeo de Estudiantes (Sevilla y Urbán, 2008). 
movilizaciones condujo a la desaparición de asambleas, manteniéndose únicamente las de la UAB y algunas de la UB. La PMDUP se sostuvo fundamentalmente por los esfuerzos de AEP y CEPC -luego $\mathrm{SEPC}^{26}$ - . De ser una gran coordinadora, esta plataforma devino en una estructura que fundamentalmente organizaba las movilizaciones anuales.

Las coordinadoras europeas se concentraron en reunir organizaciones políticas con rama estudiantil o sindicatos de estudiantes que compartían un ángulo de visión anticapitalista. Mientras tanto, en los campus universitarios no dejaban de hacerse patentes fuentes de malestar sectorial y un aparente repliegue corporativo-localista contra fuertes problemáticas: a) la introducción irregular de las automatrículas, b) los cobros abusivos y c) el nuevo régimen académico a tiempo completo de los estudiantes. Por otro lado, se reactivaron las huelgas de becarios y se crearon nuevos espacios de aglutinación de investigadores y docentes precarios ${ }^{27}$.

Al margen de una debilitada PMDUP, la jornada del 6 de Marzo de 2008 constituyó un antes y un después en términos logísticos, organizativos y discursivos. Su convocatoria en Barcelona fue apoyada por las Asambleas de la UAB, el SEPC y la pujante asamblea del Raval (UB). En la UAB surgió el Comité de Vaga (Comité de Huelga), en el cual se integraron representantes de cada asamblea de facultad. Entre ellos, había militantes de los dos sindicatos estudiantiles (SEPC y AEP) y profesores de la $\mathrm{CGT}^{28}$ y de la Asamblea contra la Precariedad. Así pues, en la convocatoria de huelga de la UAB se aunarían reivindicaciones laborales y estudiantiles, bajo un marco común de denuncia de la LOU, del Real Decreto de Grados y del EEES.

El Comité de Vaga organizó una Semana de Lucha previa, cuya acción principal fue la ocupación de la Facultad de Filosofía y Letras. La "solución" rectoral fue bloquear la interlocución y llamar a un dispositivo de antidisturbios, en lo que se conoció como "los hechos del 4 de marzo" ${ }^{29}$. La manifestación del 6 de marzo de 2008 escenificó una confluencia ejemplar de luchas en defensa del sector público con la inclusión de la denuncia de la LEC - que ya había provocado varios paros magisteriales- y la presencia de los conductores de autobuses de $\mathrm{TMB}^{30}$ en

\footnotetext{
26. Sindicat d'Estudiants dels Països Catalans, organización estudiantil de la izquierda independentista fruto de la fusión entre la CEPC i la Alternativa Estel.

27. Asamblea Contra la Precariedad en la UAB, D-Recerca en la UB y Federación de Jóvenes Investigadores, a nivel estatal.

28. Confederación General del Trabajo, sindicato libertario y anarcosindicalista con presencia en el sector de profesorado en la UAB.

29. Esta intervención produjo medio centenar de heridos, la dimisión del adjunto del Vicerrector de Estudiantes y de la Vicedecana de la Facultad de Psicología.
}

30. Transports Metropolitants de Barcelona, empresa pública metropolitana de transporte de viajeros. 
huelga. Por otra parte, uno de los efectos colaterales del éxito de la jornada fue la implosión de la PMDUP y su sustitución en el verano del 2008 por la Coordinadora d'Assemblees d'Estudiants (CAE). La CAE eliminaba la posibilidad de representación sindical y blindaba una línea discursiva conocida como "no frontal a Bolonia" (frente al "sí crítico" o al "sí con financiación").

El Comité de Vaga de la UAB se disolvió y el trabajo de las asambleas se reorientó hacia la petición de dimisión del Rector y el bloqueo institucional a la aplicación de Bolonia. Todo ello implicaría tanto el retorno activo a los órganos de gobierno ${ }^{31}$, como una acumulación de costes represivos ${ }^{32}$.En estas circunstancias, el curso 2008/2009 comenzaba con la convocatoria del 20 de noviembre, puesta de largo de la CAE. Por su parte, el SEPC había lanzado la campaña nacional "DiguEEES no"33 e impulsaba la realización de referendos en todos los campus ${ }^{34}$.

Para organizar la semana de lucha previa a la manifestación se creaba una nueva estructura de coordinación: el Comité Contra Bolonia ${ }^{35}$. El 20 de noviembre se realizaron manifestaciones en todas las capitales de provincia de los Pä̈sos Catalans ${ }^{36}$. En Barcelona, los estudiantes okuparon el Rectorado de la UB, un edificio histórico en pleno centro de Barcelona. Durante los meses de noviembre y diciembre, en la UAB y en la UB se sucedieron las okupaciones y las asambleas que solicitaban ingreso en la $\mathrm{CAE}$ no dejaban de crecer. Al margen de todo ello, las autoridades universitarias -a través de l'Associació Catalana d'Universitats Públiques (ACUP) - se negaban a paralizar el proceso de Bolonia y a realizar cualquier tipo de referéndum ${ }^{37}$. Mientras, los comunicados de apoyo a las okupaciones y en solidaridad con los expedientados, no dejaban de sucederse ${ }^{98}$. Sin embargo, la coordinación estatal no llegó a cuajar al no ser respaldada por el SEPC.

31. La "traición" del rectorado en la etapa anterior se había traducido en la política de "sillas vacías"

32. 31 expedientados, 6 expulsados y más de 30 imputaciones penales, todas ellas contra líderes estudiantiles.

33. Juego de palabras con el vocablo catalán "digues" (que en español significa "di") y el acrónimo del Espacio Europeo de Educación Superior.

34. 15.599 estudiantes (UAB, UB y UPF), de los cuáles el 93.84\% votaron a favor de paralizar la aplicación del EEES. Las elecciones a rector tuvieron menor participación (Especial Bolonya, 2009).

35. El Comité Contra Bolonya (CCB) -compuesto por una representación de cada asamblea de facultad más otra del SEPC- planteaba: a) el reconocimiento del déficit financiero de la UAB (que imposibilitaba la implementación de los nuevos planes de estudio), b) la retirada de los expedientes disciplinarios y c) la celebración de un referéndum vinculante sobre la aplicación de la reforma.

36. En Barcelona, la UAB y UB-Raval convocaron huelga.

37. Cinco rectores de universidades okupadas y que concentran el $20 \%$ del estudiantado (UB, UAB, Universidad Complutense de Madrid, Universidad de Sevilla y Universidad de Valencia) pedían ayuda al gobierno español para neutralizar al movimiento (El País, 27/11/2008).

38. Destacar a la izquierda sindical: CGT, CNT (Confederación Nacional del Trabajo), IAC (Intersindical Alternativa de Catalunya), Co.Bas (Comités de Base) y COS (Coordinadora Obrera Sindical). 
Las okupaciones de facultades de la UAB fueron las únicas que interrumpieron docencia y llegaron a entablar negociaciones políticas ${ }^{39}$. Sin embargo, los nulos resultados de las mismas cedieron la iniciativa al gobierno español. Zapatero convocó a los rectores, se desplegaron campañas mediáticas para desprestigiar al movimiento y el Ministerio de Innovación y Ciencia anunció la negociación del Estatuto del Estudiante Universitario, con interlocutores variopintos y ninguna asamblea $^{40}$. Contrariamente, la okupación del Rectorado de la UB centraba su discurso en la denuncia del proceso de reforma, en su falta de legitimidad y en la petición de diálogo.

El Comité Contra Bolonia (CCB) no contaba con asambleas masivas y en el corto periodo de un mes de intensa actividad, la falta de comunicación y transparencia informativa redundaría en una debilidad progresiva. En estas circunstancias, se propuso el desalojo voluntario de las facultades para okupar el Rectorado de la UAB, pero el periodo vacacional (Navidad) deslució la acción. Finalmente, en una rueda de prensa ante el rectorado de la UB, se escenificaba la incorporación del activismo de la UAB. El Rectorado okupado se convirtió en la "central de inteligencia” de las comisiones de la CAE, además de sede para todo tipo de encuentros de movimientos sociales ${ }^{41}$.

A mediados de febrero de 2009, el desalojo de estudiantes de la UPF del Campus de Ca l'Aranyó y la decisión del Claustro de la UB, -ignorando el resultado del referéndum- de continuar adelante con el proceso de reformas, provocaron la convocatoria por parte de la CAE de una jornada de huelga el 12 de marzo de $2009^{42}$. Una semana después, la madrugada del 18 de marzo de 2009, el desalojo violento del Rectorado okupado y las posteriores cargas policiales contra las concentraciones de protesta devinieron en disturbios por todo el centro de la ciudad y levantaron ampollas en el establishment político ${ }^{43}$. La Consellería d'Interior consideró “proporcionadas las cargas”, mientras los periódicos se llenaban de relatos de ciudadanos que habían sufrido la violencia policial y emergían los comunicados de denuncia de entidades vecinales y cívicas ${ }^{44}$.

39. Se reunió en la UAB la mesa de negociación de más alto nivel con la plana mayor del Departament d'Universitats y de la Associació Catalana d'Universitats Públiques.

40. En Catalunya el Govern organizó otra homóloga, la Taula Nacional per a la Universitat Pública.

41. Surgió la Asamblea PDI-PAS (Personal Docente Investigador-Personal de Administración y Servicios), se celebraron asambleas de los conductores de TMB, dos encuentros de estudiantes de secundaria, acogió la campaña internacional de denuncia de la represión estudiantil en Colombia, entre otras iniciativas.

42. El lema de la misma -"Per un moviment unitari contra la privatització de l'Educació Pública. Per la derogació de la LOU i la LEC"- ponía el acento en la lucha contra las privatizaciones.

43. Entre los cientos de heridos había periodistas, así como una familia con un niño de diez años.

44. Colegio de Abogados de Barcelona, Colegio de Periodistas, Federación de Asociaciones de Vecinos de Barcelona y Asociación Catalana de Defensa de los Derechos Humanos. 
El 26 de marzo de 2009, la CAE organizó una manifestación bajo el lema "El seu diàleg és la repressió: per la llibertat d'expressio" ${ }^{45}$. El alcalde de Barcelona anunció la ilegalidad del recorrido, pero la manifestación discurrió -para sorpresa de todos- por un recorrido alterno libre de policía y de disturbios, para acabar con los parlamentos de la Asamblea PDI-PAS ${ }^{46}$ y de la Asamblea de barrio de Sants ${ }^{47}$. Meses después, algunos activistas desalojados del Rectorado participaron en la okupación del $\mathrm{n}^{\circ} 17$ de la calle Casanovas de Barcelona. ¿ $\mathrm{Su}$ intención? Crear la Universitat Lliure ${ }^{48}$, "La Rimaia", desde donde vincularse a las luchas vecinales y de apoyo a los inmigrantes ${ }^{49}$.

Si bien durante el curso 2009-2010 siguieron los intentos infructuosos de paralizar el proceso de Bolonia, el desalojo del Rectorado okupado marcó el momento de reflujo del ciclo. Por otro lado, el inicio de la política de recortes sociales del gobierno Zapatero facilitaba, paradójicamente, el retorno de la derecha al poder. En cuanto a los otros movimientos sociales, las Plataformas de Afectados por la Hipotecas -surgidas en 2009 con el objetivo de frenar la oleada de desahucios desatada por la crisis- y las Mareas contra la privatización de la sanidad y la educación, anunciaban la llegada de un nuevo ciclo contra las políticas de austeridad (Fernández, 2014).

\section{1- ?, el ciclo anti-austeridad y recortes: 15M y Ley Wert}

La derrota del movimiento estudiantil catalán en su pulso contra la implantación del Plan Bolonia hizo mella en el ánimo de los activistas, al tiempo que propició que el sector más autónomo abandonara el movimiento estudiantil para ingresar en los movimientos urbano-populares de la capital catalana. Luchas como las del movimiento de okupaciones, los movimientos contra las subidas de precios de los transportes públicos, el 15M o las huelgas generales de 2012, fueron animadas y protagonizadas por la generación de activistas forjada en el movimiento contra Bolonia.

\footnotetext{
45. "Su dialogo es la represión: por la libertad de expresión"

46. La Asamblea PDI-PAS (2009) editó un libro muy recomendable sobre esa coyuntura.

47. "[...] os saludamos a todos aquéllos y aquéllas que lucháis contra el Plan Bolonia. Os queremos decir que estamos con vosotros y que los barrios son vuestra casa, que sentimos vuestra lucha como nuestra, la privatización de la universidad es una de las mil formas con las que nos están robando la vida, que nos están robando nuestros sueños, nuestras maneras de construir un presente y un futuro más allá de la miseria cotidiana que nos impone el capitalismo" (Assemblea de Barri, 2009).
}

\section{En español, Universidad Libre}

49. "La Rimaia" fue desalojada, pero una nueva okupación la reubicó en la Gran Vía. En julio de 2010 se sucedieron desalojos y nuevas okupaciones, hasta dar con un emplazamiento más duradero en la Ronda de Sant Pau donde permaneció hasta el 9 de mayo de 2012, como estandarte de los movimientos alternativos de Barcelona. 
Mientras, en la universidad, la hegemonía del SEPC era cuestionada por contra-movimientos generados por la propia institución que bebían en el caldo de cultivo de un proceso complejo. Por un lado, había cierta animadversión hacia el vanguardismo de la etapa anterior y, por el otro, se dio un cambio en el perfil social del estudiantado generado por las propias políticas de mercantilización de la educación y los incrementos del $67 \%$ en los precios de las tasas de matriculación en $2012^{50}$. La PAT (Plataforma de Afectados por las Tasas) denunciaba en un manifiesto la expulsión de miles de jóvenes de la enseñanza universitaria (PAT, 2013). Los estudiantes más afectados por la subida de las tasas son los de clase obrera o clase media-baja, la cual cosa comporta una elitización de los estudios universitarios, en especial de los de maestría (Fernández, Urbán y Sevilla, 2013).

El movimiento de los indignados se inició con la toma simultánea de las principales plazas de las ciudades de todo el Estado español el 15 de mayo de 2011, en medio de la campaña electoral de las elecciones municipales y autonómicas. En el 15M, se plasmó de forma sorprendente el hartazgo de amplios sectores sociales, especialmente jóvenes, ante las políticas de ajuste en los países del Sur de Europa como única respuesta a la crisis económica mundial iniciada en 2008. La novedad es que a una minoría de militantes y activistas de los ciclos de lucha de los 90 y 2000, se sumaron, en una estrategia de desobediencia civil, miles de ciudadanos que desbordaban a los propios convocantes. La participación de universitarios, por ejemplo, iba mucho más allá de las organizaciones estudiantiles tradicionales y de las de nuevo cuño. Una nueva generación militante se incorporaba con un discurso de indignación ante la situación económica, la falta de perspectivas y la inoperancia del poder político. El señalamiento de políticos y banqueros como culpables de la situación constituía un aterrizaje y una simplificación necesaria de las "antiguas" consignas contra la globalización capitalista (Calle, 2012 e Ibarra, 2012).

Las subidas de tasas, el surgimiento de un nuevo ciclo de movilizaciones entorno al $15 \mathrm{M}$ a partir de las Mareas contra la privatización de la Educación y la Sanidad o el crecimiento de las luchas por una vivienda digna a través de las Plataformas de Afectados por las Hipotecas, fueron el contexto de la masiva respuesta de todo el sector educativo (incluido el universitario) contra la reforma en clave neo-conservadora y centralista de la Ley Orgánica de Educación (LOMCE o Ley Wert). El anteproyecto, la discusión en las cortes y su posterior aprobación fueron recibidas por diversas jornadas unitarias de huelga de todo el sector educativo en los cursos 2012-2013 y 2013-2014. En el

50. En el curso 2012-2013 las tasas aumentaron un 67\%. El precio de la matrícula universitaria pública osciló entre 1566 y 2372 euros. Respecto al año 2001 los aumentos oscilan entre el 84 y el 103\% (El Triangle, 9/10/2012). El número de estudiantes en peligro de abandonar la universidad por no poder pagar las tasas de matriculación ascendió 30.000 en el conjunto del Estado (Silió y Vallespín, 2013 ). 
caso catalán, las huelgas también protestaban contra el despliegue de la LEC, a través de organismos de viejo tipo como la PMDUP, el MUCE (Marc Unitari de la Comunitat Eductiva), SOM Escola (en defensa de la lengua catalana) y de nuevo tipo como las Asambleas y Mareas Amarillas ${ }^{51}$.

El movimiento estudiantil universitario participó -pero no protagonizó- en este último ciclo de movilización todavía vigente, donde destacaron más los estudiantes de secundaria, los maestros y profesores de enseñanza primaria y media, así como las luchas contra la precariedad laboral de los profesores universitarios jóvenes. Aun así, la PMDUP convocó de nuevo manifestaciones y jornadas de lucha. En el marco de una de ellas, la CAF impulsó una nueva okupación del rectorado de la UAB de un mes de duración entre el 17 de abril y el 17 de mayo de 2013. La okupación quedó aislada del ciclo de movilización y no fue seguida de okupaciones homólogas en otras universidades.

Una cuestión que plantea esta última etapa es si en el Estado español y en Catalunya nos hallamos ante un sólo ciclo u onda larga de 20 años (19942014, con diversas etapas o sub-ciclos) o si existen, como parecen argumentar algunos autores (Antentas y Vivas, 2012), dos ciclos diferenciados: una contra la globalización neoliberal -que finalizaría con el movimiento antiguerra-y otro contra las políticas de austeridad como respuesta a la crisis económica de 2008, que empezaría en 2011 con el 15M y la Primavera Árabe. Siguiendo a Herreros (2004) y en términos de ciclos de movilización de la historia contemporánea, no existen elementos de diferenciación profunda entre los nuevos movimientos globales y los movimientos contra el austericidio, sino que más bien éstos últimos -como el 15M, las PAH o las Mareas- suponen el aterrizaje práctico y local de las consignas y demandas de los primeros. Al señalar a la clase política y a los banqueros como responsables de la crisis, se concreta mucho más cuál es el ejecutor de las políticas dictadas por las lejanas instituciones y organismos transnacionales. Las propuestas de procesos constituyentes o la negativa al pago de la deuda, también aterrizan a escenarios plausibles los objetivos genéricos de justicia social y democracia participativa planteados por los nuevos movimientos globales en las etapas precedentes.

51. Por el color de las camisetas que con el lema "SOS Educación Pública" portaban manifestantes y huelguistas. 


\section{Conclusiones}

El movimiento estudiantil catalán se ha retroalimentado del ciclo contra la globalización neoliberal, en una dinámica de alianzas crecientes con sectores educativos (laborales y docentes) y movimientos sociales. En la tabla 1 se presenta la correspondencia cronológica de los nuevos movimientos globales con el movimiento estudiantil.

Tabla 1. Ciclos de movilización de los nuevos movimientos globales y del movimiento estudiantil en Catalunya.

\begin{tabular}{|c|c|}
\hline Nuevos Movimientos Globales. & Movimiento estudiantil \\
\hline $1^{\text {a } \text { etapa. Gestación 1994-1999. }}$ & 1993-1999. Movilizaciones antitasas \\
\hline $\begin{array}{c}2^{\text {a }} \text { etapa. Eclosión, 2000-2002. } \\
\text { Movimiento anti-guerra, 2003-2004 }\end{array}$ & $\begin{array}{c}\text { 2000-2004. Del No a la la Lou al No a la } \\
\text { Guerra }\end{array}$ \\
\hline $\begin{array}{c}\text { 3a etapa. Transformaciones. Luchas } \\
\text { contra la crisis (vivienda) 2005-2010. }\end{array}$ & 2005- 2010. Ciclo anti-Bolonia (EEES). \\
\hline $\begin{array}{c}\text { 4a etapa. Ciclo anti-austeridad. 2011-? } \\
\text { 15M, PAH, Mareas. }\end{array}$ & $\begin{array}{c}\text { 2011- ? Contra los recortes en } \\
\text { educación y la Ley Wert. }\end{array}$ \\
\hline
\end{tabular}

Fuente: Elaboración propia.

Comentando la Tabla 1, y como se ha analizado en los resultados de este artículo, se concluye que existe un desarrollo paralelo de ambos movimientos, con algunos contrastes, que a continuación se sintetizan. En la primera etapa de gestación de los nuevos movimientos globales, el movimiento estudiantil reaparece como sujeto político, se construye hacia dentro y establece los marcos organizativos y discursivos que desarrollará en los siguientes periodos. En la segunda etapa, la de eclosión de los nuevos movimientos sociales, el movimiento estudiantil adopta una dimensión global con su participación en los Foros Sociales y en las movilizaciones contra la guerra de Irak. Así mismo, esta segunda etapa es la que gozará de las manifestaciones más masivas al coincidir con el momento álgido de la antiglobalización y con un ciclo de protestas local contra el gobierno del PP. La tercera etapa es la que presenta ciertos contrastes entre ambos movimientos que no descartan en absoluto su integración en el mismo ciclo de luchas en términos históricos. Así cierto reflujo o transformación hacia movimientos más concretos, como el de vivienda, coincide con la internacionalización definitiva de las luchas estudiantiles que con el ciclo anti-Bolonia asumen al completo el marco discursivo contra el neoliberalismo que los nuevos movimientos globales venían planteando. Finalmente, la cuarta etapa supone, para los nuevos movimientos globales el aterrizaje práctico de las consignas y objetivos de la antiglobalización, 
y para el movimiento estudiantil universitario la certeza de que aislado no puede enfrentar los retos que plantea el neoliberalismo y, por tanto, la urgencia de continuar con la confluencia con las Mareas contra los recortes en Sanidad y Educación en sentido amplio.

El movimiento estudiantil, igual que los nuevos movimientos globales, ha sabido a lo largo de este ciclo de luchas responder a sus retos: construir marcos flexibles, pero estables de organización; reforzar las redes internacionales de resistencia a los procesos de mercantilización de la enseñanza y tejer alianzas con otros colectivos en lucha vinculados a la enseñanza (profesores precarios, maestros y personal de administración y servicios).

En cuanto al análisis de contenido del discurso que se ha realizado en esta investigación, se concluye que el movimiento estudiantil ha sabido plantear ante la opinión pública unos marcos de significado alternos a los de las autoridades universitarias. Así, ante la supuesta modernización que implicaba el proceso de Bolonia, el movimiento ha denunciado la mercantilización de la enseñanza; ante las políticas de austeridad presupuestaria y su supuesta inevitabilidad, el movimiento ha planteado los recortes en educación como retrocesos en los derechos sociales y democráticos de los ciudadanos llevados a cabo por una clase política sumisa a los intereses de bancos y transnacionales.

Sin embargo, en algunas fases cruciales de este ciclo la estrategia de desgaste y acoso institucional mediante la represión al movimiento, incentivó derivas internas vanguardistas, subordinando la dinámica de la lucha estudiantil al metabolismo del activismo. Estas circunstancias explican por qué un movimiento, que en 2008-2009 tuvo en jaque al gobierno, no consiguió ni la moratoria del proceso de Bolonia, ni la readmisión de las personas expulsadas, ni la derogación de la LOU o el advenimiento de su posterior versión neoconservadora, la LOMCE. De cualquier manera, es ineludible destacar la riqueza y diversidad de acciones y de herramientas que produjo el movimiento en una explosión de creatividad propia de un ciclo de movilización. Además, las formas organizativas horizontales que ensayó en busca de una democracia de base se encuentran en la génesis de experiencias como el $15 \mathrm{M}$, que apuntan a futuros escenarios de transformación social y política profunda en el Estado español. Cabe destacar entre ellas la pervivencia de la CAF en la UAB, o la eficacia democrática de otros organismos de coordinación como la PMDUP o la CAE.

Desde 1993 a 2013 se perciben continuidades en el movimiento estudiantil catalán tanto en lo organizativo como en lo discursivo. Esto convierte a este periodo histórico en un largo ciclo de luchas estudiantiles contra los efectos del neoliberalismo en el modelo de universidad. Mientras tanto...cada año la universidad se llena de nuevos estudiantes y -como dicen los zapatistas- los rebeldes se buscan y cuando se encuentran, todo es posible. 


\section{Referencias}

Antentas, J.M. (2001). Les resistències a l'Europa neoliberal. En Emergències, quaderns de formació per a la transformació social, 1, 5-13.

Antentas, J.M. y Vivas, E. (2012). El 15M y la razón indignada. En Papeles de relaciones ecosociales y cambio global, 118, 129-140.

Assemblea de Barri (2009). Manifest de suport als estudiants antibolonia, 26 de marzo, Barcelona: octavilla.

Assemblea Pdi-Pas (2009). La cara fosca del Pla Bolonya. Contra la Universitat S.A. En defensa de la universitat pública, Barcelona: Edicions Bellaterra.

Bonal, X. y Verger, A. (2013). L'agenda de la política educativa a Catalunya: un anàlisi de les opcions de govern (2011-2013). Informes Breus, 45, Fundació Jaume Bofill, Barcelona.

Brockett, C. (1991). The Structure of Political Oportunities and Peasent Mobilization in Central America. Comparative Politics, 53, 253-274.

Calle, A. (2005). Los nuevos movimientos globales: hacia la radicalidad democrática, Madrid, España: Popular.

Calle, A. (2012). 15M. Más allá de la lírica. Las naturalezas (ya no tan subterráneas) del 15M. Viento Sur, 123, 61-69.

Camargo, R. (diciembre de 2001). La LOU y nosotras(os), que la odiamos tanto. Viento Sur, 60, Madrid, España.

Carreras, J., C. Sevilla, y Urbán, M. (2006): Euro-universidad. Mito y realidad del proceso de Bolonia, Madrid, España: Icaria Más Madera.

CEPC (2006). CEPC. Cinc anys en marxa. Construint alternatives des de les aules. Precedents de la CEPC, 25 anys de lluita estudiantil, Barcelona:

Coordiandora d'Estudiants dels Països Catalans (CEPC).

$58 \mathrm{i}+$ (2009). Especial Bolonia, Recuperado de http://especialbolonya. wordpress.com/2009/03/02/analisi-referendums/, febrero de 2010

Colectivo Maldeojo (2001). Estudiantes, antiestudiantes, policía, prensa y poder, Madrid, España: Traficantes de sueños.

El Mundo (2000). Unos 11.000 alumnos salen a la calle contra el Informe Bricall. Recuperado de http://www.elmundo.es/2000/03/24/ sociedad/24No079.html. 
El Mundo (2001). Especial: "Las aulas se levantan". Recuperado de http://www.elmundo.es/especiales/2001/10/sociedad/educacion/ calendario.html,noviembre de 2009.

El País (2008). Los rectores de Madrid se manifestaran por primera vez en defensa de la universidad. Recuperado de http://elpais.com/elpais/2008/11/27/ actualidad/1227777423_850215.html.

El Triangle (2012). Actualitat al carrer. Recuperado de http://www.eltriangle. eu/client/print/print_notice.php? IDN=31954.

Especial Bolonya (2009). Referèndums sobre Bolonya: Representativitat i democràcia als Campus Universitaris. Recuperado de http:// especialbolonya.wordpress.com/2009/03/02/analisi-referendums/

Etxezarreta, M. (2001). Algunos rasgos de la globalización. En R. Fernández et al., Globalización capitalista. Luchas y resistencias. Barcelona-Bilbao: Virus-Baladre.

Fernández, J., Urbán M. y Sevilla, C. (Coords.) (2013). De la nueva miseria. La universidad en crisis y la nueva rebelión estudiantil, Madrid, España: Akal.

Fernández, J. (2014). El estudio del impacto de los movimientos sociales: el caso del movimiento estudiantil anti-bolonia. Oximora, 4, 1-22. Revista Internacional de Ética y Política

García, R. y Fernández, J. (2008). Movimiento universitario en Bizkaia contra la invasión de Irak. En Asamblea de Ciencias Sociales por una Universidad Crítica. Movimientos estudiantiles: resistir, imaginar, crear en la universidad, Donostia: Gakoa Liburuak.

González, R. y Barranco, O. (2007). Construyendo alternativas frente a la globalización neoliberal. Resistencias juveniles en Catalunya, Revista de Estudios de Juventud, 76, 267-285.

González, R. (2011). Estudiantes contra la globalización capitalista. El caso de Catalunya. En P. Ibarra y M. Cortina (Eds.). Recuperando la Radicalidad: un encuentro entorno al análisis político crítico, Barcelona, España: Hacer Editorial.

González, R. (2013). Ley Wert. Una embestida contra la lengua catalana. Viento Sur.info. Recuperado de (http://www.vientosur.info/spip. php?article7565).

Herreros, T. (2004). Moviments socials i cicles de protesta: el cicle de protesta del capitalisme global, 1994-2003. Ponencia en el VIII Congreso español de sociologia. FES, Alicante. 
Ibarra, P. (2012). 15M. Más allá de la lírica. Una aproximación comparativa. Viento Sur, 123, 45-53.

Iglesias, P. (2005). 13M POSSE. Comunicación, movimiento global y autonomía. [Ponencia]. VII Congreso de la Asociación Española de Ciencia Política y de la Administración, Madrid, Septiembre de 2005.

Kitschelt, H. (1986). Political opportunity structures and political protest: antinuclear movements in four democracies. British Journal of Political Science, 16, 57-85.

La Vanguardia. (2001). Del Castillo promete diálogo con el sector para reformar la secundaria. Los claustros de las universidades de Barcelona y Girona debaten esta semana la Ley de Universidades. Recuperado de http://hemeroteca.lavanguardia.com/ preview/2001/11/18/pagina37/34203987/pdf.html.

León, I. y Sanllorente, I. (2008). II. Retrospectiva de las movilizaciones universitarias frente a la Ley Orgánica Universitaria (2000 a 2002). En Asamblea de Ciencias Sociales por una Universidad Crítica. Movimientos estudiantiles: resistir, imaginar, crear en la universidad, Donostia: Gakoa Liburuak.

McAdam, D. (1998). Orígenes conceptuales, problemas actuales y direcciones futuras. En P. Ibarra y B. Tejerina (Eds.). Los movimientos sociales. Transformaciones políticas y cambio cultural. Madrid, España: Trotta.

Navarrete, M. (1995). El movimiento estudiantil en España. De 1965 a 1985. Revista Acciones e Investigaciones Sociales, 3, 121-136.

Okupem Les Ones (2009). El Pròleg, memòries d'un any de lluites contra el Pla Bolonya [Documental sobre las movilizaciones 2008-2009]. Barcelona: okupem les ones.

PAT (2013). Plataforma de Afectados por las Tasas. Nos están desahuciando de la Educación. Manifiesto. Recuperado de http:// afectadasporlastasas.net/manifiesto/.

Rodríguez, E. (2003). La universidad y su crítica. Movimiento estudiantil, reforma universitaria y mercado de trabajo. Logos, Anales del Seminario de Metafísica, 36, 49-63.

Rosas, M. (2001). Plebeyas Batallas. La huelga en la universidad, México, D.F.: Era.

Salazar, C. (2012). ¿Hemos estudiado por encima de las necesidades del mercado? Viento Sur.info.Recuperado de http://www.vientosur.info/spip. php?article7301. 
Sampedro, V. (2005). 13-M Multitudes on line, Madrid, España: Los libros de la catarata.

Sevilla, C. (2006). Tesis sobre la Universidad y el movimiento estudiantil, Viento Sur, 87, 47-54.

Sevilla, C. (2010). La fábrica del conocimiento. La universidad-empresa en la producción flexible, Barcelona, España: El Viejo Topo.

Sevilla, C. y Urbán, M. (2008). Tesis sobre la Universidad y el movimiento estudiantil. En Asamblea de Ciencias Sociales por una Universidad Crítica. Movimientos estudiantiles: resistir, imaginar, crear en la universidad. Donostia: Gakoa Liburuak.

Silió, E. y Vallespín, I. (6 de junio de 2013). Más de 30.000 estudiantes al borde de la expulsión por impago. En El País. Recuperado de http://sociedad. elpais.com/sociedad/2013/06/17/actualidad/1371499455_460313. htm17/06/2014.

Tarrow, S. (1997): El poder en movimiento: los movimientos sociales, la acción colectiva y política. Madrid, España: Alianza.

Tilly, C. (1991). Grandes estructuras, procesos amplios, comparaciones enormes, Madrid, España: Alianza.

UAB INFORMA'T (diciembre de 2001). Butlletí Informatiu de les Assemblees $d^{\prime}$ Estudiants, UAB: Manuscrito.

Vaquero, C. (2004). El movimiento estudiantil universitario. De la Ley de Autonomía Universitaria (1979) a la Ley Orgánica de Universidades (2001). Mientras Tanto, 91-92, 155-166.

Verger, A (2005). Que entenem per investigació activista? En Col lectiu Investigacció, Recerca Activista i Moviments Socials. Barcelona, España: El Viejo Topo. 